\title{
THE FLOW OF BLOOD IN THE CORONARY ARTERIES IN PATHOLOGICAL HEARTS
}

\author{
By WILLIAM B. KOUNTZ AND JOHN R. SMITH \\ (From the Department of Internal Medicine, Washington University School of Medicine, and \\ the Barnes Hospital, St. Lonis)
}

(Received for publication September 30, 1937)

Investigation of functional changes in pathological human hearts has been fraught with many difficulties. Particularly has this been true in the important question of coronary flow in disease of the coronary arteries and in conditions of myocardial weakness or hypertrophy. With animal experiments, it has not been possible to simulate closely the pathological changes of humans. Sudden partial ligation of coronary arteries resembles only to a slight extent the gradual sclerosis and later occlusion which occurs in the arteries of man. The blood flow and the state of the myocardium in such artificial states cannot be accurately compared with the changes which have slowly developed in the relatively long span of human life.

Revival of pathological human hearts according to methods which we have previously described appeared to open a new approach to the functional study of heart disease. The way, however, was not easy and many difficulties and interfering factors were encountered.

In reviving the human heart the ideal method of study is with a heart lung preparation in which the beating muscle can be caused to do a fixed amount of work and in which the right heart can perform approximately its normal function. Less favorable for study is the revival maintained by constant perfusion of the coronary arteries in an isolated heart.

In hearts of patients dying of cardiac insufficiency, revival is relatively difficult. The time after death is a far more important factor than in those from individuals who have been dead from other causes. Even when the preparation is made very promptly, careful massage is necessary to establish cardiac activity. Some hearts are difficult to revive with the best of technique. The degree of revival varies and may be insufficient to sustain pulmonary circulation. Occasionally one obtains a heart which responds well but because of edema of the lungs, or other pulmonary complications, one is unable to establish satisfactorily blood flow through the lungs. In only two instances in patients dying from heart failure were we able to make a heart lung preparation. It was, therefore, necessary to use the perfused isolated heart in studying coronary flow in pathological conditions.

One of the incentives for undertaking this work was the hope that it might furnish important information concerning the influence of the physical state of the heart on coronary inflow. Experiments on dogs' hearts have established that there is definite variation in flow during the normal cardiac cycle. There has been, however, much dispute $(1,2)$ concerning both the nature and cause of the variation. Some $(3,4)$ have believed that the systolic phase of the heart beat leads to a diminution in the coronary flow, while others $(5,6)$ find that the decreased flow is at times synchronous with diastole. The variations have been attributed by some authors to changes in the physical state of the heart (7). For experimental study of such a problem, human hearts are superior to those of dogs. They are less irritable and, under usual circumstances, will not fibrillate so readily. With its greater muscle mass, the human heart is not weakened to the same degree when asphyxia is used as an experimental method for dilatation. Evidence concerning the relationship between coronary flow and the physical state of the heart can be accurately obtained only with heart lung preparations of which two, from pathological hearts, could be compared with normal hearts previously reported.

\section{METHODS}

The methods for perfusion and heart lung preparations have been described in detail in previous publications. The most essential feature is a cannula which permits the development of a normal or high coronary artery pressure without great distention of the weakened ventricles. Important parts of the apparatus include (1) 
a Starling heart lung apparatus, (2) a reservoir connected with a coronary cannula and controlled by gravity, (3) a second system consisting of a reservoir and connecting cannula in the coronary arteries, (4) a system by which one may slowly increase the load in the left ventricle and shift it to the heart lung apparatus, and (5) an apparatus for recording coronary flow, size of the heart, volume and output. The coronary flow was measured through the cannulae in the coronary arteries which were perfused by blood passing from a reservoir. In the top of the reservoir was placed a so-called hot wire which measured the amount of air replacing the blood flowing from it into the coronary system. The volume of the heart could be measured by recording the distribution of blood in various systems at any particular moment as well as by an oncometer over it. The pressures in the cannula of the coronary arteries could be maintained at any desired level; usually $120 \mathrm{~mm}$. $\mathrm{Hg}$ was the point at which the gauges were set. The pressure in the ventricle, on the other hand, could be varied according to the contractility of the heart. In hearts whose power to contract was reduced, pressure was necessarily established at about $20 \mathrm{~mm}$. $\mathrm{Hg}$ but, as the ability of the heart to increase its load improved during the progress of the experiment, the pressure in the left ventricle was increased. One was seldom able to increase the pressure to $120 \mathrm{~mm}$. $\mathrm{Hg}$ without dilating the heart. About $80 \mathrm{~mm}$. seemed to be the maximum pressure in perfusion experiments; whereas in heart lung experiments it could be increased to $120 \mathrm{~mm}$. $\mathrm{Hg}$.

We have, by such methods, revived and studied 15 hearts of individuals who died of chronic cardiac failure. In all instances the actual heart weight and ventricular weights were determined, after the method of Herrmann and Wilson (11).

While the coronary flow in the untreated heart was the first consideration, the action of drugs on coronary flow and heart volume was studied also.

After the flow in perfused hearts had been recorded, drugs were injected directly into the coronary circulation. The results, some of which have been recorded previously (8), were compared with similar experiences in hearts of non-cardiac patients.

The influence of the diastolic volume of the heart was studied in the heart lung preparations. The procedure consisted in adjusting the apparatus so that the diastolic volume and the coronary flow would be as constant as possible. Drugs, some of which were known to influence diastolic volume, were injected (11). Asphyxia was then produced. This was accomplished by shutting off the coronary flow. After an interval, depending upon the diastolic state, the drugs were re-administered and the effect was compared with the results before dilatation. The influence of three groups of drugs was considered. The first group consisted of digitalis, epinephrin, and pituitrin, and each was chosen because it decreased the cardiac volume. In the second group were histamine and sodium nitrite which were used because they increase the diastolic volume and also increase the coronary artery inflow in the normal heart. The third group con-

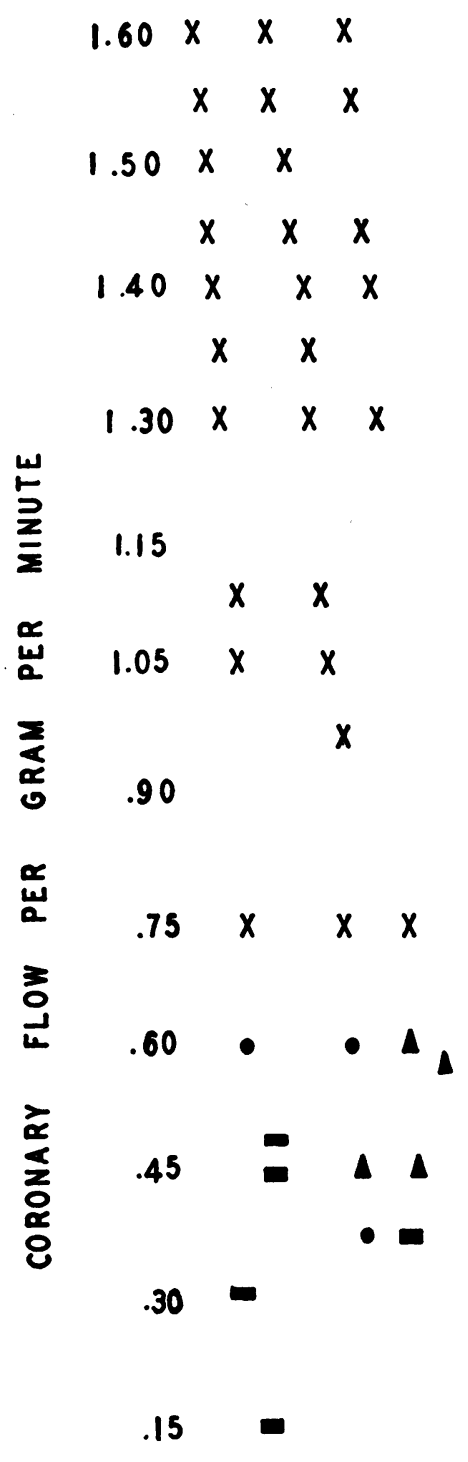

Fig. 1

The average coronary flow for normal hearts is about $1.4 \mathrm{cc}$. per gram per minute in perfused heart. In those which have been decompensated, coronary flow ranges between 0.15 and $0.6 \mathrm{cc}$. per gram of heart muscle per minute of the coronary artery pressure in heart muscles.

- Arteriosclerotic heart disease

Rheumatic heart disease (Right coronary flow)

Syphilitic heart disease

$\times$ Patient dying without history of decompensation.

sisted of xanthine compounds such as theobromine, sodium salicylate and caffein sodium benzoate which, when injected into the perfusing solution, dilate the coronary arteries and coronary rings with only a minimal influence on cardiac volume. 
RESULTS

Coronary flow per gram of heart beat per minute was less in the hearts which were perfused than in those that had been made into a heart lung preparation, owing to the fact that the right ventricle was functioning better in conditions of heart lung preparation than it was when the heart was perfused. This, of course, makes it impossible to compare the results obtained under the two sets of experiments. Normal hearts have been perfused, however, many times and comparison with normal coronary artery flow under identical conditions could be made.

The total coronary flow of a normal heart, whose weight was around 300 grams and whose rate was 64 beats per minute, was found to average, when perfused, about 421 cc. per minute which represented about 1.4 cc. per gram per minute. In adults' hearts without coronary disease, there was some variation; but with a pressure of $80 \mathrm{~mm}$. $\mathrm{Hg}$, the flow seldom dropped below $0.9 \mathrm{cc}$. or rose above $1.5 \mathrm{cc}$. In younger individuals the flow often exceeded this figure and sometimes equalled $1.6 \mathrm{cc}$. per gram of heart muscle per minute. In the hearts of individuals dying of heart disease the total coronary artery flow was often greater, especially in hearts where a marked hypertrophy had taken place, but when reduced to the terms of unit weight, it was found to fall below the level of $0.75 \mathrm{cc}$. per gram of heart per minute. In subjects who have had a history of heart failure the flow ranged from 0.15 to $0.6 \mathrm{cc}$. per gram per minute. It is interesting that the three individuals with a coronary flow of 0.75 cc. gave a definite cardiac history, although the cause of death was not cardiac.

The lowest figures were encountered in the arteriosclerotic hearts. In luetic heart disease, although the total flow was usually increased, the flow per unit weight was greatly diminished. The hearts of four cases of syphilitic aortic insufficiency which averaged 790 grams in weight were found to have an average coronary flow of 410 cc. or 0.52 per gram of heart muscle per minute.

The effect of increased pressure upon the flow into the coronary arteries in these hearts is of interest. When the coronary flow with a gradually increasing pressure is taken in normal and hypertrophied hearts, it is found that the flow in the normal ones for a time parallels the increased pressure.

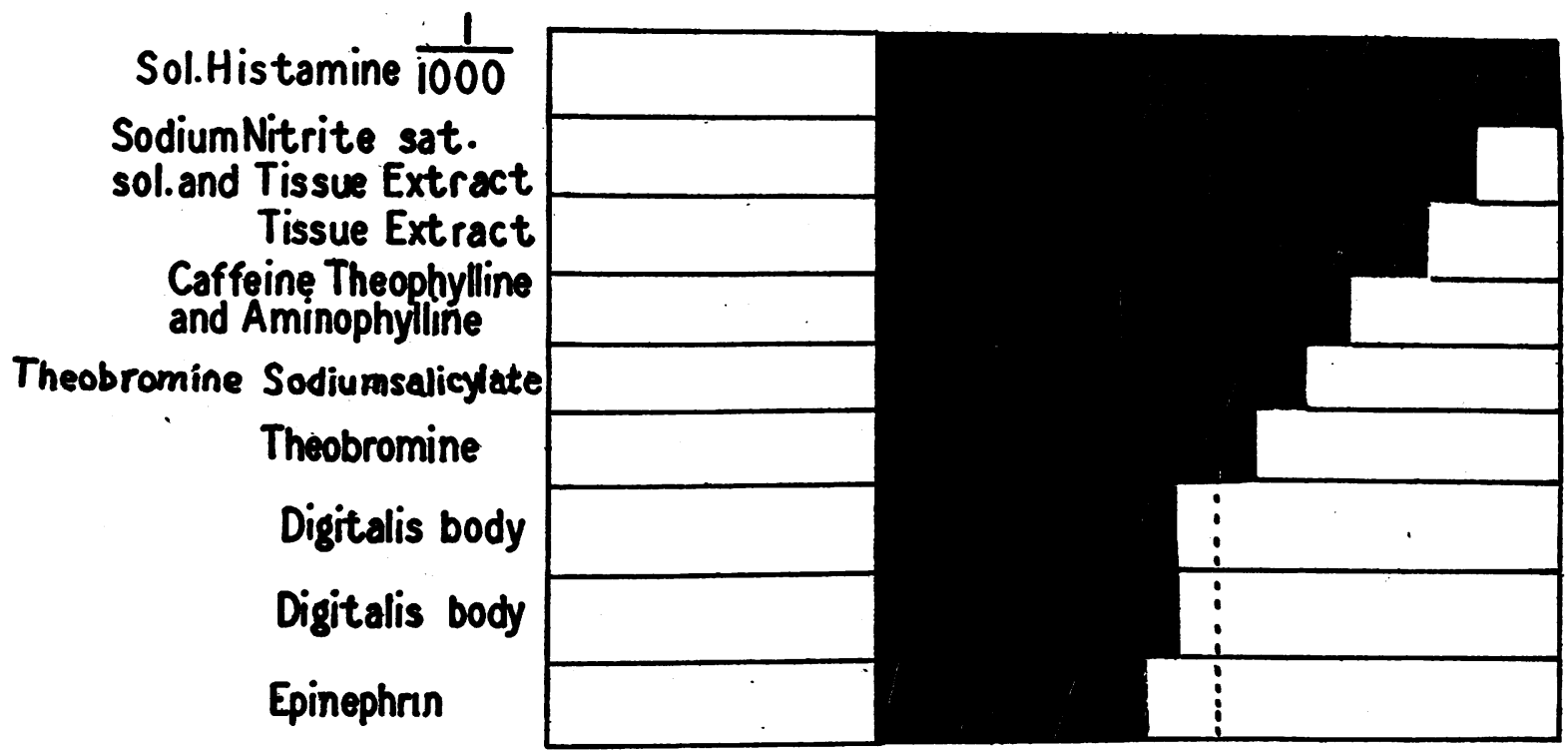

Fig. 2. Noryal Beating Heare. The Efrects of Drugs on Coronary Flow of Umdinated Perfused Hearts.

The light area, to the left of the figure, indicates the average flow in the coronary arteries per gram of heart muscle per minute; whereas, the shaded area indicates the flow after the addition of the drug to the perfusing flow. The coronary perfusion and ventricular pressures were the same in each experiment. Caffeine theophyllin and aminophylline and theobromine sodium salicylate were in acid solution. 
In hypertrophied hearts, the response of the coronary artery inflow to an increase in perfusion pressure is practically negative.

The rheumatic heart with a mitral lesion is rather more difficult to study than those of simple hypertrophy, since in this condition only the right ventricle is involved. Theoretically, one should compare only the right ventricular weight with the blood flow in the right coronary artery. In many instances, however, vessels other than the right coronary supply blood to the muscle of the correct ratio of coronary inflow to heart muscle. By the former calculation the flow was found to average $0.45 \mathrm{cc}$. per gram of right ventricular weight per minute. By using the entire flow in a total estimate of heart weight, the minute flow was found to be well above $0.35 \mathrm{cc}$. per gram.

The action of drugs, especially those which change the diastolic volume of the heart, is found to vary, depending primarily upon the physical state of the organ.

The dilated hearts (those in which the diastolic

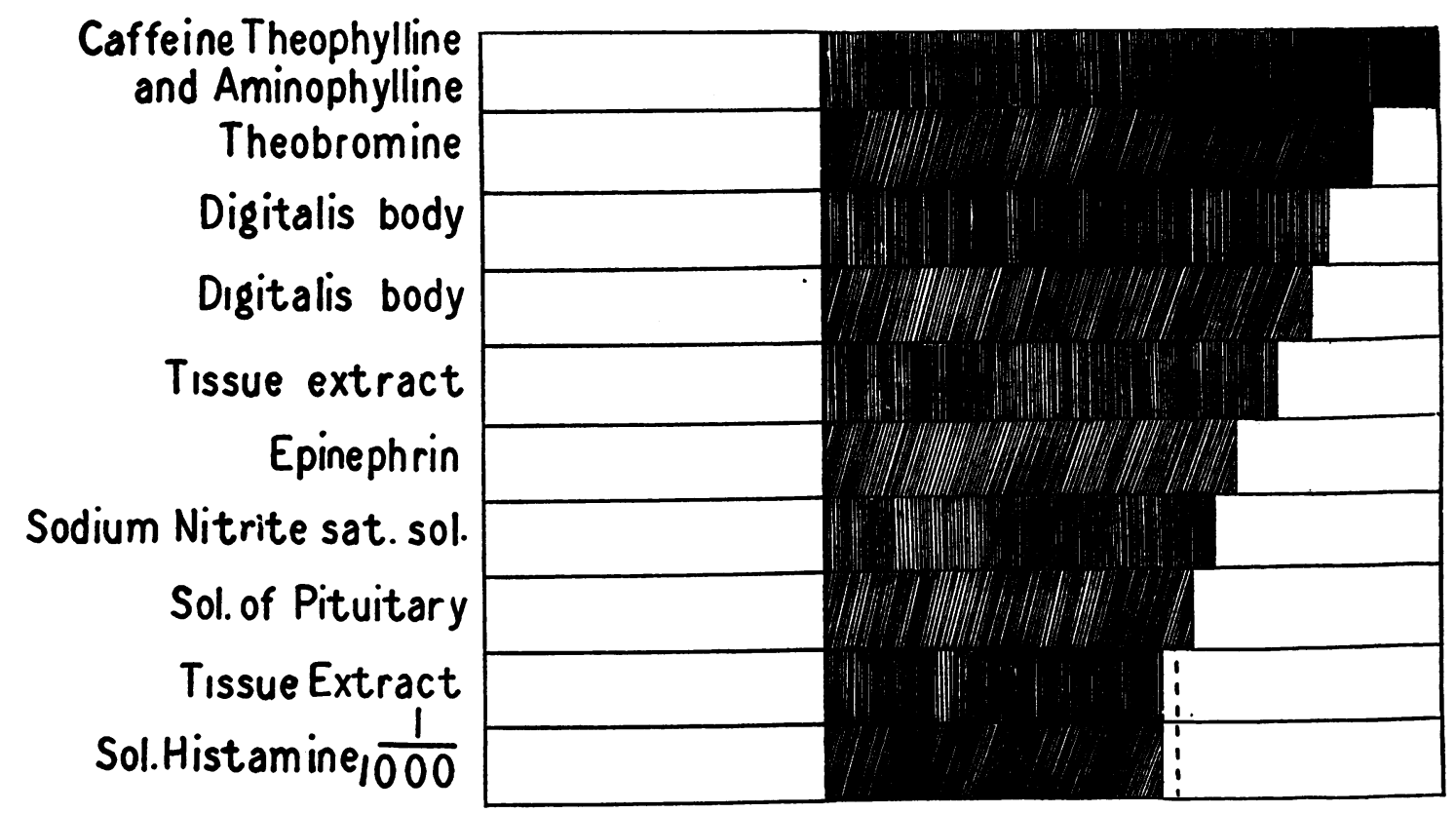

Fig. 3. Dilated Human Hearts. Effects of Drugs on the Coronary Artery flow in the Same Hearts as Used for Figure 2, After the Diastolic Volume Had Been Increased by Temporarily Reducing the Coronary Artery Flow.

The drugs were injected into the perfusing solution at the dose as previously used. When the two figures are compared, a reversal in action of some of the drugs may be noted. Perfusion and ventricular pressures were the same in each experiment. Caffeine theophylline and aminophylline and theobromine were in acid solution.

right ventricle, thus rendering it difficult to estimate the ratio of coronary flow to grams of heart muscle to this chamber. One, however, could obtain an estimate by such a procedure. In addition, we checked the figure by weighing the right ventricle and establishing an estimated weight of the left side. We then took the total coronary artery inflow which we had measured and determined the ratio of coronary flow to the heart weight. Since the results by the two methods are similar, we assume that the figure is close to the volume had been increased) revealed two important characteristics. It was found that those drugs which diminish the diastolic volume increase the inflow, while those which increase the diastolic volume of the heart diminish coronary artery flow. These results are exactly opposite to those obtained on hearts with normal diastolic volume. The second characteristic was revealed by detailed analysis of the coronary artery inflow by means of the hot wire. This was a shift in the time relationship of the maximum coronary artery in- 
flow during diastole and systole. In the dilated hearts the maximum inflow occurs during systole when the organ approaches nearest to its normal diastolic state.

Increasing dilatation of a heart with a constant work factor and constant coronary artery pressure

TABLE I

Measurements of coronary flow in normal and dilated hearts

\begin{tabular}{c|c|c|c|c|c}
\hline & \multicolumn{2}{|c|}{ Normal hearts } & \multicolumn{3}{|c}{ Dilated hearts } \\
\cline { 2 - 6 } $\begin{array}{c}\text { Perfusion } \\
\text { pressure }\end{array}$ & $\begin{array}{c}\text { Diastolic } \\
\text { size * }\end{array}$ & $\begin{array}{c}\text { Coronary } \\
\text { flow } t\end{array}$ & $\begin{array}{c}\text { Perfusion } \\
\text { pressure }\end{array}$ & $\begin{array}{c}\text { Diastolic } \\
\text { size * }\end{array}$ & $\begin{array}{c}\text { Coronary } \\
\text { flow } \dagger\end{array}$ \\
\hline$m m . B 8$ & & & $\begin{array}{c}m m . H g \\
\text { mg }\end{array}$ & & \\
120 & 575 & 200 & 120 & 890 & 217 \\
124 & 542 & 270 & 117 & 784 & 176 \\
116 & 525 & 225 & 122 & 818 & 190 \\
140 & 636 & 289 & 131 & 925 & 205 \\
\hline
\end{tabular}

* Cubic centimeters of water displaced.

† Cubic centimeters of blood per minute.

progressively reduced the coronary inflow. Table I reveals the definite influence that change in the diastolic volume has upon the flow.

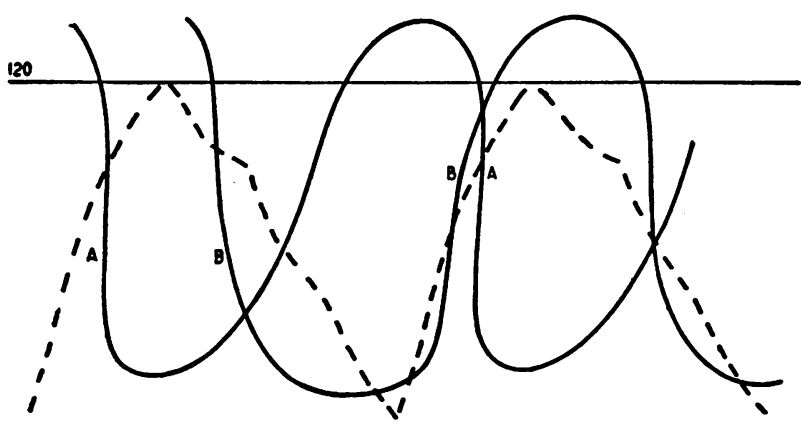

FIG. 4.

The solid lines are superimposed tracings of curves. Line $A$ represents the inflow in a heart which is in about a normal physical state, while $B$ represents the inflow after it has been dilated. The down stroke of the curve represents a swing of the galvanometer string to the base line and indicates diminution in the coronary flow; whereas, the up stroke represents a swing from the base line and indicates an increase of flow into the coronary artery. The reduction in the coronary flow occurs in a normal heart chiefly during systole, or during the period that the pressure in the aorta is on the increase, while the reduction in coronary flow in dilated hearts occurs chiefly during the period of diastole or when the pressure in the aorta is reduced.

\section{DISCUSSION}

This work indicates that hearts fail in chronic cardiac disease because of a limited coronary artery flow. This may result from either of two factors, increased muscle mass or constriction of the coronary arteries. In either case the minute blood flow under the conditions of the experiment was reduced to well below $0.75 \mathrm{cc}$. per gram of heart muscle. Actually the flow varied between $0.15 \mathrm{cc}$. and $0.6 \mathrm{cc}$. It is perhaps noteworthy that the lower values always appeared in older individuals and in association with arteriosclerotic heart disease. This fact suggests, perhaps, that lessened physical activity either in patients, or in old age, enables the heart to withstand the reduced coronary artery flow. It would appear, however, that with any blood flow below the level of $0.6 \mathrm{cc}$. per gram per minute, the function of the heart is endangered.

One of the primary requisites in treatment of the failing heart would seem to be an increase in the coronary flow. The experiments on the effect of drugs clearly demonstrate the necessity of considering the physical state of the heart in the selection of therapeutic agents. Hearts with increase in diastolic volume react differently than normal hearts. Drugs such as histamine and nitrites which, under normal conditions, increase coronary flow, will diminish it when the heart is dilated. Digitalis and epinephrin, on the other hand, which reduce coronary flow in normal hearts, augment the flow of hearts in which the diastolic volume is increased.

Such experimental evidence is in accord with clinical observations. It has long been known that nitrites are ineffective or even detrimental in dilated decompensated hearts. Digitalis which is so helpful in cardiac dilatation diminishes the minute volume output of normal hearts.

Explanation of these results cannot be offered with certainty. Ten Cate (10) has suggested that chemical changes in the heart muscle, such as increase in acidity, may influence the action of drugs. In the asphyxia which is used in our experiments to produce dilatation such a factor might be of importance. It appears to us more probable, however, that the change is chiefly owing to variations in the physical state of the heart. Since Anrep et al. (1) have shown that even the 
normal systolic contraction tends to obstruct the blood flow in the coronary arteries, it does not seem improbable that abnormal dilatation made by stretching and narrowing of the blood vessels causes a decrease in the coronary artery flow. It can readily be seen that drugs which dilate the heart or increase its relaxation might augment stretching of the coronary vessels, while those which tend to decrease the diastolic volume might permit the vessels to return to a more nearly normal state.

Of interest in this regard are the observations on coronary flow during systole and diastole. In normal hearts, measurements of flow by the hot wire method disclosed an increase with diastole and diminution with each contraction of the heart. This result was also obtained by Anrep in a study of the hearts of normal dogs. As has been seen, the opposite is true when the diastolic volume of the heart is increased. Drugs which in their action tended to increase the systolic phase and thereby to diminish the size of the heart would tend to increase coronary flow.

The influence of deranged metabolism which does exist in the hearts may well be secondary to the limited circulation.

\section{SUMMARY AND CONCLUSIONS}

1. In hearts of patients who have died of heart failure, the coronary blood flow is diminished either absolutely, as in diseases of the coronary arteries and in dilatation of the heart, or relatively, as in hypertrophy.

2. Under the conditions of these experiments, it appears that any rate of flow less than 0.75 per gram of heart muscle per minute endangers the function of the heart.

3. Either hypertrophy or dilatation reduces the coronary flow per gram of heart muscle. In hypertrophy this may be accounted for by increase in muscle mass. In dilatation, lengthening and stretching of coronary vessels may be the chief factor.

4. In dilated hearts, the coronary flow is increased during systole and diminished during diastole; a result which is exactly opposite to that found in hearts of normal diastolic volume.
5. In the dilated heart, drugs which tend to decrease the diastolic volume increase the coronary flow, while those which augment diastolic volume diminish the flow. This action also is directly opposite to the phenomena observed in normal undilated hearts. It suggests that the physical state of the heart, and particularly the degree of dilatation, must be considered in the selection of drugs for the treatment of cardiac disease.

The authors wish to express their appreciation to the following for assistance in this work: Doctors E. F. Pierson, Karl Koenig, M. Prinzmetal and Walter Seibert.

\section{BIBLIOGRAPHY}

1. Anrep, G. V., Cruickshank, E. W. H., Downing, A. C., and Subba Rau, A., The coronary circulation in relation to the cardiac cycle. Heart, 1927-29, 14, 111.

2. Hochrein, M., and Keller, J., Untersuchungen am Koronarsystem. Arch. f. exper. Path. u. Pharmakol., 1931, 159, 300.

3. Anrep, G. V., Blalock, A., and Hammouda, M., The distribution of the blood in the coronary blood vessels. J. Physiol., 1929, 67, 87.

4. Anrep, G. V., Davis, J. C., and Volhard, E., The effect of pulse pressure upon the coronary blood flow. J. Physiol., 1931, 73, 405.

5. Anrep, G. V., and Von Saalfeld, E., The effect of the cardiac contraction upon the coronary flow. J. Physiol., 1933, 79, 317.

6. Hochrein, M., Der Coronarkreislauf : Physiologie, Pathologie, Therapie. Julius Springer, Berlin, 1932.

7. Stehle, R. L., and Melville, K. I., The influence of the heart beat upon the flow of blood into the coronary arteries. J. Pharmacol. and Exper. Therap., 1932, 46, 477.

8. Wiggers, C. J. In Levy, R. L., Diseases of the Coronary Arteries and Cardiac Pain. Macmillan Company, New York, 1936, p. 89.

9. Kountz, Wm. B., Studies on the coronary arteries of the human heart. J. Pharmacol. and Exper. Therap., 1932, 45, 65.

10. Ten Cate, J., L'influence du Calcium dans l'Action du Sympathique sur le Coeur de Grenouille. Arch. Neerl., de physiol., 1926, 10, 498.

11. Herrmann, G. R., and Wilson, F. N., Ventricular hypertrophy. A comparison of electrocardiographic and postmortem observations. Heart, 1921, 9, 91.

12. Bodo, Richard, The effect of the "heart tonics" and other drugs upon the heart tone and coronary circulation. J. Physiol., 1928, 64, 365. 